Supplement of Biogeosciences Discuss., 12, 11833-11861, 2015

http://www.biogeosciences-discuss.net/12/11833/2015/

doi:10.5194/bgd-12-11833-2015-supplement

(C) Author(s) 2015. CC Attribution 3.0 License.

(c) (1)

Supplement of

\title{
Modelling anomalies in the spring and autumn land surface phenology of the European forest
}

\author{
V. F. Rodriguez-Galiano et al. \\ Correspondence to: V. F. Rodriguez-Galiano (vrgaliano@gmail.com)
}

The copyright of individual parts of the supplement might differ from the CC-BY 3.0 licence. 


\section{Supporting information}

Table S1. Random Forest models for anomalies in spring phenology. TX, TN and TG: maximum, minimum and average temperature, respectively; PP: precipitation; SIS: surface incoming shortwave radiation; DAL: surface radiation daylight; GDD: growing degree days; CHIL: chilling requirements; FF, LF and PF: first, last and period of freeze, respectively.

\begin{tabular}{|c|c|c|c|c|c|c|c|c|c|c|c|c|}
\hline & & & & & & Models & & & & & & Ps- \\
\hline GDD03 & MTG3 & MTX3 & MTN3 & CHIL3 & $\mathrm{F}$ & CRR3 & MRR3 & CSIS3 & MSIS3 & CDAL3 & MDAL3 & 0.58 \\
\hline GDD03 & MTG9 & MTX9 & MTN9 & CHIL3 & $\mathrm{F}$ & CRR3 & MRR9 & CSIS3 & MSIS9 & CDAL3 & MDAL9 & 0.61 \\
\hline ĠDD53 & MTG3 & MTX3 & MTN3 & C̈HIL3 & $\overline{\mathrm{F}}$ & ĊRR3 & MRR3 & ĊSIS3 & MSIS3 & ĊDAL3 & MDAL3 & 0.57 \\
\hline ĜDD53 & MTTG9 & MTX9 & MTN9 & CेHIL3 & $\overline{\mathrm{F}}$ & ĈRR3 & MRR9 & ĊSIS3 & MSIS9 & C̄DAL3 & M̄DAL9 & 0.61 \\
\hline ĠDD09 & MTG3 & МТХ3 & MTN3 & ĊHIL9 & $\overline{\mathrm{F}}$ & ĊRR9 & MRR3 & ĊSIS9 & MSIS3 & ĊDAL9 & MDAL3 & 0.58 \\
\hline ĠDD09 & M̄TG9 & MTX9 & MTN9 & C̈HIL9 & $\overline{\mathrm{F}}$ & C̄RR9 & MRR9 & ĊSIS9 & MSIS9 & ĊDAL9 & MDDAL9 & 0.60 \\
\hline ḠDD59 & MTG3 & MTX3 & MTNN3 & ĊHIL9 & $\overline{\mathrm{F}}$ & C̄RR9 & M̂RR3 & ĊSIS9 & MSIS3 & C̄DAL9 & M̄DAL3 & 0.59 \\
\hline ĠDD59 & MTG9 & MTX9 & MTN9 & C̈HIL9 & $\overline{\mathrm{F}}$ & C̈RR9 & MRR9 & ĊSIS9 & MSIS9 & ĊDAL9 & MDAL9 & 0.61 \\
\hline ĜDD03 & MTTG3 & MิTX3 & MTN3 & ĈHIL3 & $\overline{\mathrm{F}}$ & ĈRR3 & MRR3 & & & & & 0.54 \\
\hline ĠDD03 & MTG9 & MTX9 & MTN9 & ĊHIL3 & $\overline{\mathrm{F}}$ & ĊRR3 & MRR9 & & & & & 0.57 \\
\hline ḠDD53 & MTG3 & MTX3 & MTN3 & C̈HIL3 & $\overline{\mathrm{F}}$ & ĊRR3 & MRR3 & & & & & 0.54 \\
\hline ḠDD53 & MTG9 & МТX9 & MTN9 & ĊHIL3 & $\overline{\mathrm{F}}$ & ĊRR3 & MRR9 & & & & & 0.57 \\
\hline ĜDD09 & MTG3 & MTXX & MTN3 & ĊHIL9 & $\overline{\mathrm{F}}$ & C̄RR9 & MRR3 & & & & & 0.54 \\
\hline ĜDD09 & MTG9 & MTX9 & MTN9 & ĈHIL9 & $\overline{\mathrm{F}}$ & ĈRR9 & MRR9 & & & & & 0.53 \\
\hline ĠDD59 & MTG3 & МТX3 & MTN3 & ĊHIL9 & $\overline{\mathrm{F}}$ & C̈RR9 & MRR3 & & & & & 0.55 \\
\hline ĠDD59 & MTG9 & MTX9 & MTN9 & ĊHIL9 & $\overline{\mathrm{F}}$ & CRR9 & MRR9 & & & & & 0.55 \\
\hline ĠDD03 & MTG3 & MTX3 & MTN3 & ĊHIL3 & $\overline{\mathrm{F}}$ & & & & & & & 0.54 \\
\hline ĠDD03 & MTG9 & MTX9 & MTN9 & ĊHIL3 & $\overline{\mathrm{F}}$ & & & & & & & 0.56 \\
\hline ĜDD53 & MTTG3 & MTTX3 & MTTN3 & ĊHIL3 & $\overline{\mathrm{F}}$ & & & & & & & 0.53 \\
\hline ḠDD53 & MTG9 & MTX9 & MTN9 & ĊHIL3 & $\overline{\mathrm{F}}$ & & & & & & & 0.56 \\
\hline ĜDD09 & MTG3 & М̈TX3 & MTN3 & ĊHIL9 & $\overline{\mathrm{F}}$ & & & & & & & 0.52 \\
\hline ĠDD09 & MTG9 & MTX9 & MTN9 & ĊHIL9 & $\overline{\mathrm{F}}$ & & & & & & & 0.52 \\
\hline ḠDD59 & MTG3 & МТХ3 & MTN3 & ĊHIL9 & $\overline{\mathrm{F}}$ & & & & & & & 0.52 \\
\hline ḠDD59 & MTG9 & MTX9 & MTNN9 & ĊHIL9 & $\overline{\mathrm{F}}$ & & & & & & & 0.54 \\
\hline ḠDD03 & MTG3 & MTX3 & MTN3 & C̈HIL30 & & & & & & & & 0.48 \\
\hline ḠDD03 & MTG9 & MTX9 & MTN9 & CHIL30 & & & & & & & & 0.49 \\
\hline ḠDD53 & MTG3 & MTX3 & MTN3 & CHIL30 & & & & & & & & 0.48 \\
\hline ĠDD53 & MTG9 & MTX9 & MTN9 & CHIL30 & & & & & & & & 0.49 \\
\hline ĠDD09 & MTG3 & MTX3 & MTN3 & CHIL90 & & & & & & & & 0.49 \\
\hline ĠDD09 & MTG9 & MTX9 & MTN9 & CHIL90 & & & & & & & & 0.45 \\
\hline ḠDD59 & MTG3 & MTX3 & MTN3 & CHIL90 & & & & & & & & 0.48 \\
\hline ĠDD59 & MTG9 & MTX9 & MTN9 & CHIL90 & & & & & & & & 0.47 \\
\hline ĠDD03 & MTG3 & М̈TX3 & MTN3 & & & & & & & & & 0.41 \\
\hline ĠDD03 & MTG9 & MTX9 & MTN9 & & & & & & & & & 0.38 \\
\hline ḠDD53 & MTG3 & МТХЗ & MTN3 & & & & & & & & & 0.41 \\
\hline ḠDD53 & MTG9 & MTX9 & MTN9 & & & & & & & & & 0.37 \\
\hline ĠDD09 & MTG3 & МтX3 & MTN3 & & & & & & & & & 0.43 \\
\hline ḠDD09 & MTTG9 & MTX9 & MTNN9 & & & & & & & & & 0.37 \\
\hline ĠDD59 & MTG3 & М̈TX3 & MTN3 & & & & & & & & & 0.42 \\
\hline ḠDD59 & MTG9 & MTX9 & MTN9 & & & & & & & & & 0.40 \\
\hline
\end{tabular}

Table S2. Random Forest models for anomalies in autumn phenology. TX, TN and TG: maximum, minimum and average temperature, respectively; PP: precipitation; SIS: surface incoming shortwave radiation; DAL: surface radiation daylight; GDD: growing degree days; CHIL: chilling requirements; FF, LF and PF: first, last and period of freeze, respectively.

\begin{tabular}{|c|c|c|c|c|c|c|c|c|c|c|c|c|c|c|}
\hline & & & & & & Models & & & & & & & & Ps-R ${ }^{2}$ \\
\hline GDD030 & MTG30 & МТX30 & MTN30 & CRR30 & MRR30 & CSIS30 & MSIS30 & CDAL30 & MDAL30 & FF & $\mathrm{FF}$ & $\mathrm{LF}$ & $\mathrm{PF}$ & 0.78 \\
\hline GDD030 & MTG90 & MTX90 & MTN90 & CRR30 & MRR90 & CSIS30 & MSIS90 & CDAL30 & MDAL90 & $\mathrm{FF}$ & $\mathrm{FF}$ & $\mathrm{LF}$ & $\mathrm{PF}$ & 0.81 \\
\hline GDD530 & MTG30 & МТХ30 & MTN30 & CRR30 & MRR30 & CSIS30 & MSIS30 & CDAL30 & MDAL30 & FF & FF & $\mathrm{LF}$ & $\mathrm{PF}$ & 0.78 \\
\hline GDD530 & MTG90 & MTX90 & MTN90 & CRR30 & MRR90 & CSIS30 & MSIS90 & CDAL30 & MDAL90 & $\mathrm{FF}$ & FF & $\mathrm{LF}$ & $\mathrm{PF}$ & 0.81 \\
\hline GDD090 & MTG30 & MTX30 & MTN30 & CRR90 & MRR30 & CSIS90 & MSIS30 & CDAL90 & MDAL30 & FF & $\mathrm{FF}$ & $\mathrm{LF}$ & $\mathrm{PF}$ & 0.81 \\
\hline
\end{tabular}




\begin{tabular}{|c|c|c|c|c|c|c|c|c|c|c|c|c|c|c|}
\hline GDD090 & MTG90 & MTX90 & MTN90 & CRR90 & MRR90 & CSIS90 & MSIS90 & CDAL90 & MDAL90 & FF & $\mathrm{FF}$ & LF & $\mathrm{PF}$ & 0.80 \\
\hline GDD590 & MTG30 & MTX30 & MTN30 & CRR90 & MRR30 & CSIS90 & MSIS30 & CDAL90 & MDAL30 & FF & $\mathrm{FF}$ & LF & $\mathrm{PF}$ & 0.81 \\
\hline GDD590 & MTG90 & MTX90 & MTN90 & CRR90 & MRR90 & CSIS90 & MSIS90 & CDAL90 & MDAL90 & FF & $\mathrm{FF}$ & LF & $\mathrm{PF}$ & 0.79 \\
\hline GDD030 & MTG30 & MTX30 & MTN30 & CRR30 & MRR30 & CSIS30 & MSIS30 & CDAL30 & MDAL30 & & & & & 0.74 \\
\hline GDD030 & MTG90 & MTX90 & MTN90 & CRR30 & MRR90 & CSIS30 & MSIS90 & CDAL30 & MDAL90 & & & & & 0.81 \\
\hline GDD530 & MTG30 & MTX30 & MTN30 & CRR30 & MRR30 & CSIS30 & MSIS30 & CDAL30 & MDAL30 & & & & & 0.75 \\
\hline GDD530 & MTG90 & MTX90 & MTN90 & CRR30 & MRR90 & CSIS30 & MSIS90 & CDAL30 & MDAL90 & & & & & 0.81 \\
\hline GDD090 & MTG90 & MTX90 & MTN90 & CRR90 & MRR90 & CSIS90 & MSIS90 & CDAL90 & MDAL90 & & & & & 0.79 \\
\hline GDD090 & MTG30 & MTX30 & MTN30 & CRR90 & MRR30 & CSIS90 & MSIS30 & CDAL90 & MDAL30 & & & & & 0.80 \\
\hline GDD590 & MTG90 & MTX90 & MTN90 & CRR90 & MRR90 & CSIS90 & MSIS90 & CDAL90 & MDAL90 & & & & & 0.79 \\
\hline GDD590 & MTG30 & MTX30 & MTN30 & CRR90 & MRR30 & CSIS90 & MSIS30 & CDAL90 & MDAL30 & & & & & 0.79 \\
\hline GDD030 & MTG30 & MTX30 & MTN30 & CRR30 & MRR30 & $\mathrm{FF}$ & $\mathrm{FF}$ & LF & $\mathrm{PF}$ & & & & & 0.72 \\
\hline GDD030 & MTG90 & MTX90 & MTN90 & CRR30 & MRR90 & $\mathrm{FF}$ & FF & LF & $\mathrm{PF}$ & & & & & 0.77 \\
\hline GDD530 & MTG30 & MTX30 & MTN30 & CRR30 & MRR30 & $\mathrm{FF}$ & FF & LF & $\mathrm{PF}$ & & & & & 0.74 \\
\hline GDD530 & MTG90 & MTX90 & MTN90 & CRR30 & MRR90 & FF & $\mathrm{FF}$ & LF & $\mathrm{PF}$ & & & & & 0.77 \\
\hline GDD090 & MTG30 & MTX30 & MTN30 & CRR90 & MRR30 & FF & $\mathrm{FF}$ & LF & $\mathrm{PF}$ & & & & & 0.76 \\
\hline GDD090 & MTG90 & MTX90 & MTN90 & CRR90 & MRR90 & $\mathrm{FF}$ & FF & LF & $\mathrm{PF}$ & & & & & 0.73 \\
\hline GDD590 & MTG30 & MTX30 & MTN30 & CRR90 & MRR30 & $\mathrm{FF}$ & $\mathrm{FF}$ & LF & $\mathrm{PF}$ & & & & & 0.76 \\
\hline GDD590 & MTG90 & MTX90 & MTN90 & CRR90 & MRR90 & $\mathrm{FF}$ & FF & LF & $\mathrm{PF}$ & & & & & 0.73 \\
\hline GDD030 & MTG30 & MTX30 & MTN30 & CSIS30 & MSIS30 & CDAL30 & MDAL30 & & & & & & & 0.72 \\
\hline GDD030 & MTG90 & MTX90 & MTN90 & CSIS30 & MSIS90 & CDAL30 & MDAL90 & & & & & & & 0.78 \\
\hline GDD530 & MTG30 & MTX30 & MTN30 & CSIS30 & MSIS30 & CDAL30 & MDAL30 & & & & & & & 0.73 \\
\hline GDD530 & MTG90 & MTX90 & MTN90 & CSIS30 & MSIS90 & CDAL30 & MDAL90 & & & & & & & 0.77 \\
\hline GDD090 & MTG90 & MTX90 & MTN90 & CSIS90 & MSIS90 & CDAL90 & MDAL90 & & & & & & & 0.77 \\
\hline GDD090 & MTG30 & MTX30 & MTN30 & CSIS90 & MSIS30 & CDAL90 & MDAL30 & & & & & & & 0.77 \\
\hline GDD590 & MTG90 & MTX90 & MTN90 & CSIS90 & MSIS90 & CDAL90 & MDAL90 & & & & & & & 0.77 \\
\hline GDD590 & MTG30 & MTX30 & MTN30 & CSIS90 & MSIS30 & CDAL90 & MDAL30 & & & & & & & 0.76 \\
\hline GDD030 & MTG30 & MTX30 & MTN30 & $\mathrm{FF}$ & $\mathrm{FF}$ & LF & PF & & & & & & & 0.71 \\
\hline GDD030 & MTG90 & MTX90 & MTN90 & $\mathrm{FF}$ & $\mathrm{FF}$ & LF & PF & & & & & & & 0.73 \\
\hline GDD530 & MTG30 & MTX30 & MTN30 & $\mathrm{FF}$ & $\mathrm{FF}$ & LF & PF & & & & & & & 0.72 \\
\hline GDD530 & MTG90 & MTX90 & MTN90 & $\mathrm{FF}$ & $\mathrm{FF}$ & LF & $\mathrm{PF}$ & & & & & & & 0.72 \\
\hline GDD090 & MTG30 & MTX30 & MTN30 & $\mathrm{FF}$ & $\mathrm{FF}$ & LF & PF & & & & & & & 0.72 \\
\hline GDD090 & MTG90 & MTX90 & MTN90 & $\mathrm{FF}$ & $\mathrm{FF}$ & LF & PF & & & & & & & 0.70 \\
\hline GDD590 & MTG30 & MTX30 & MTN30 & $\mathrm{FF}$ & $\mathrm{FF}$ & LF & PF & & & & & & & 0.71 \\
\hline GDD590 & MTG90 & MTX90 & MTN90 & $\mathrm{FF}$ & $\mathrm{FF}$ & LF & PF & & & & & & & 0.70 \\
\hline GDD030 & MTG30 & MTX30 & MTN30 & CRR30 & MRR30 & & & & & & & & & 0.66 \\
\hline GDD030 & MTG90 & MTX90 & MTN90 & CRR30 & MRR90 & & & & & & & & & 0.75 \\
\hline GDD530 & MTG30 & MTX30 & MTN30 & CRR30 & MRR30 & & & & & & & & & 0.68 \\
\hline GDD530 & MTG90 & MTX90 & MTN90 & CRR30 & MRR90 & & & & & & & & & 0.75 \\
\hline GDD090 & MTG90 & MTX90 & MTN90 & CRR90 & MRR90 & & & & & & & & & 0.69 \\
\hline GDD090 & MTG30 & MTX30 & MTN30 & CRR90 & MRR30 & & & & & & & & & 0.73 \\
\hline GDD590 & MTG90 & MTX90 & MTN90 & CRR90 & MRR90 & & & & & & & & & 0.70 \\
\hline GDD590 & MTG30 & МTX30 & MTN30 & CRR90 & MRR30 & & & & & & & & & 0.73 \\
\hline
\end{tabular}


Table S3. Random Forest models for anomalies in autumn phenology considering spring anomalies as a predictor. TX, TN and TG: maximum, minimum and average temperature, respectively; PP:

precipitation; SIS: surface incoming shortwave radiation; DAL: surface radiation daylight; GDD: growing degree days; CHIL: chilling requirements; FF, LF and PF: first, last and period of freeze, respectively.

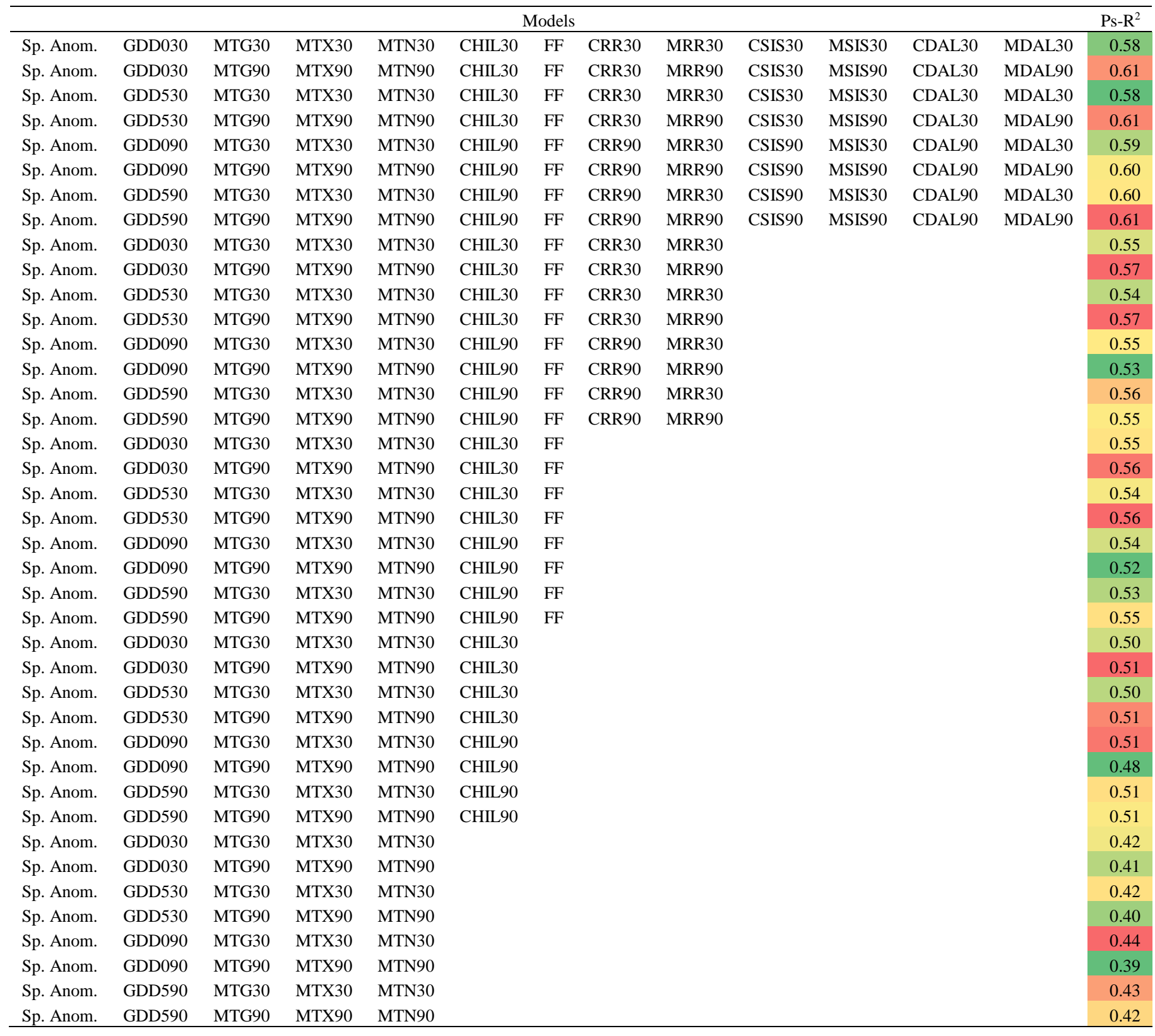

